\title{
Erratum to: Matter, Places and Persons in Cahokian Depositional Acts
}

\author{
Sarah E. Baires ${ }^{1} \cdot$ Melissa R. Baltus ${ }^{2}$ \\ Published online: 15 February 2017 \\ (C) Springer Science+Business Media New York 2017
}

\section{Erratum to: J Archaeol Method Theory DOI 10.1007/s10816-016-9304-0}

This erratum is to correct Table 2 originally published in the online version of our article Matter, Places, and Persons in Cahokian Depositional Acts doi: 10.1007/s10816-0169304-0. At the time of publication, Table 2 was missing the citations column citing the sources for the presented data. Here we include the corrected table (with the addition of a citations column) and would like to acknowledge the Illinois State Archaeological Survey for permissions to use unpublished data from the following sites: Goose Ditch, Baby Moon, Fingers South, and Curtiss Steinberg. We also include the references associated with the table.

\section{References}

Baltus, Melissa R. 2014 Transforming Material Relationships: 13th Century Revitalization of Cahokian Religious-Politics. Ph.D. Dissertation, Department of Anthropology, University of Illinois at Urbana-Champaign.

Betzenhauser, Allen M. 2011 Creating the Cahokian Community: The Power of Place in Early Mississippian Sociopolitical Dynamics. Ph.D. Dissertation, Department of Anthropology, University of Illinois at Urbana-Champaign.

The online version of the original article can be found at http://dx.doi.org/10.1007/s10816-016-9304-0.

Sarah E. Baires

bairess@easternct.edu

1 Eastern Connecticut State University, 421 Webb Hall 83 Windham Street, Willimantic, CT 06226, USA

2 University of Toledo, University Hall 2520, 2801 W. Bancroft Street, Toledo, OH 43606, USA 
Table 2 "Cached" tools from Terminal Late Woodland and Mississippian sites in the American Bottom region

\begin{tabular}{|c|c|c|c|}
\hline Phase & Site & “Cached" Objects & Citation \\
\hline Late Woodland & Range & 2 limestone hoes & Kelly et al. 1987 \\
\hline \multirow{9}{*}{$\begin{array}{l}\text { Terminal Late } \\
\text { Woodland }\end{array}$} & BBB Motor & celt & Emerson and Jackson 1984 \\
\hline & Range & 7 Mill Creek hoes & Kelly et al. 1990 \\
\hline & & shale hoe & \\
\hline & & Burlington hoe & \\
\hline & & 4 gouges & \\
\hline & & 15 celts & \\
\hline & & 10 adzes & \\
\hline & & 15 discoidals & \\
\hline & Washausen & 2 celts & Betzenhauser 2011 \\
\hline \multirow[t]{12}{*}{ Lohmann } & Turner & sandstone abrader & Milner 1983 \\
\hline & & sandstone metate & \\
\hline & & shell hoe & \\
\hline & & chert hoe fragment & \\
\hline & & chert cores & \\
\hline & Range & $\begin{array}{l}\text { Mill Creek hoe } \\
\text { discoidal }\end{array}$ & Hanenberger 2003 \\
\hline & Goose Ditch & biface & ISAS $^{*}$ \\
\hline & & abrader & \\
\hline & & hammerstone & \\
\hline & Lohmann & adze & Esarey and Pauketat 1992 \\
\hline & & 4 celts & \\
\hline & Emerald & Mill Creek hoe & $\begin{array}{l}\text { T. Pauketat, personal } \\
\text { communication }\end{array}$ \\
\hline \multirow[t]{3}{*}{ Lohmann/Stirling } & Baby Moon & hoe & ISAS $^{*}$ \\
\hline & Goose Ditch & celt & ISAS $^{*}$ \\
\hline & & 2 adzes & \\
\hline \multirow[t]{12}{*}{ Stirling } & Turner & 2 Mill Creek hoes & Milner 1983 \\
\hline & & microdrill core & \\
\hline & & large utilized flake & \\
\hline & & sandstone abrader & \\
\hline & & $\begin{array}{l}\text { Mill Creek Ramey knife } \\
\text { fragment }\end{array}$ & \\
\hline & & celt & \\
\hline & Robert Schneider & chert cores & Finney and Fortier 1985 \\
\hline & & hammerstone & \\
\hline & & 3 grinding stones & \\
\hline & & shell tempered bowl & \\
\hline & $\begin{array}{l}\text { Old Edwardsville } \\
\text { Road }\end{array}$ & $\begin{array}{l}\text { Mill Creek hoe } \\
\text { celt fragment }\end{array}$ & Jackson and Millhouse 2003 \\
\hline & Goose Ditch & point & ISAS $^{*}$ \\
\hline
\end{tabular}


Table 2 (continued)

\begin{tabular}{|c|c|c|c|}
\hline Phase & Site & "Cached" Objects & Citation \\
\hline & Fingers South & hoe/celt & ISAS $^{*}$ \\
\hline & & hoe blade(s) & \\
\hline & Fingers West & Powell Plain jar & Kelly 1995 \\
\hline & Labras Lake & Ramey knife & Yerkes 1987 \\
\hline & & celt & \\
\hline & Sponemann & 3 hoes & Jackson et al. 1992 \\
\hline & & metate & \\
\hline & Lohmann & hoe fragment & Esarey and Pauketat 1992 \\
\hline & $\begin{array}{l}\text { Cahokia - } \\
\text { Kunnemann } \\
\text { Mound }\end{array}$ & 2 Mill Creek hoes & Pauketat 1993 \\
\hline & Cahokia - ICT-II & cores & Collins 1990 \\
\hline & & Mill Creek hoe & \\
\hline \multirow[t]{4}{*}{ Stirling/Moorehead } & Turner & 2 Mill Creek hoes & Milner 1983 \\
\hline & & celt fragment & \\
\hline & Olin & $\begin{array}{l}\text { Crescent Hills Burlington } \\
\text { adze }\end{array}$ & Baltus 2014 \\
\hline & & Burlington pick & \\
\hline \multirow[t]{10}{*}{ Moorehead } & Curtiss Steinberg & 3 celts & ISAS $^{*}$ \\
\hline & & 2 Ramey knives & \\
\hline & & pottery trowel & \\
\hline & & biface & \\
\hline & & hoe & \\
\hline & Fingers South & discoidal & ISAS $^{*}$ \\
\hline & & Ramey knife & \\
\hline & & 2 hoes & \\
\hline & Julien & 4 Mill Creek hoes & Milner 1984b \\
\hline & & 4 Ramey knives & \\
\hline \multirow[t]{6}{*}{ Sand Prairie } & Hawkins Hollow & 3 adzes & Jackson 2015 \\
\hline & & 4 celts & \\
\hline & & celt preform & \\
\hline & & 2 Mill Creek hoes & \\
\hline & & 2 Limestone hoes & \\
\hline & & fine-grained Mill Creek spud & \\
\hline
\end{tabular}

\footnotetext{
* Unpublished data used with permission from the Illinois State Archaeological Survey
}

Collins, James M. 1990 Archaeology of the Cahokia Mounds ICT-II: Site Structure. Illinois Cultural Resources Study No. 10. Illinois Historic Preservation Agency, Springfield.

Emerson, Thomas E., and Douglas K. Jackson 1984 The BBB Motor Site. American Bottom Archaeology FAI-270 Site Reports Vol. 6. University of Illinois Press, Urbana. 
Esarey, Duane, and Timothy R. Pauketat 1992 The Lohmann Site (11S49): An Early Mississippian Center in the American Bottom. American Bottom Archaeology, FAI270 Report No. 1. University of Illinois Press, Urbana.

Finney, Fred A. and Andrew C. Fortier 1985 Carbon Dioxide (11MO594) and the Robert Schneider (11MS1177) Sites: Late Woodland, Emergent Mississippian, and Mississippian Occupations. American Bottom Archaeology FAI-270 Site Reports Vol. 11. University of Illinois Press, Urbana.

Hanenberger, Ned H. 2003 The Range Site 3: Mississippian and Oneota Occupations. Transportation Archaeological Research Report Volume 17. Illinois Transportation Archaeological Research Program, Champaign, IL.

Jackson, D.K. 2015 Hawkins Hollow: A Late Mississippian Household in the American Bottom. Illinois State Archaeological Survey Research Reports, Number 33. Illinois State Archaeological Survey, Champaign, IL.

Jackson, D.K., Andrew C. Fortier, and Joyce A. Williams 1992 The Sponemann Site 2 (11-MS-517): the Mississippian and Oneota Occupations. American Bottom Archaeology, FAI-270 Site Report Vol. 24. University of Illinois Press, Urbana.

Jackson, Douglas K. and Philip G. Millhouse 2003 The Vaughn Branch and Old Edwardsville Road Sites: Late Stirling and Early Moorehead Phase Mississippian Occupations in the Northern American Bottom. Transportation Archaeological Research Reports No. 16. Illinois Transportation Archaeological Research Program. University of Illinois at Urbana-Champaign.

Kelly, John E., Andrew C. Fortier, Steven J. Ozuk, and Joyce A. Williams 1987 The Range Site: Archaic through Late Woodland Occupations. American Bottom Archaeology FAI-270 Site Reports Volume 16. University of Illinois Press, Urbana.

1995 The Fingers and Curtiss Steinberg Road Sites: Two Stirling Phase Mississippian Farmsteads in the Goose Lake Locality. Transportation Archaeological Research Report No. 1. Illinois Transportation Archaeological Research Program. University of Illinois at Urbana Champaign.

Milner, George 1983 The Turner and DeMange Sites. American Bottom Archaeology FAI-270 Reports Vol. 4. University of Illinois Press, Urbana.

1984b The Julien Site. American Bottom Archaeology FAI-270 Site Reports Vo. 7. University of Illinois Press, Urbana.

Pauketat, Timothy R. 1993 Temples for Cahokia Lords: Preston Holder's 1955-1956 Excavations of Kunnemann Mound. Memoirs of the Museum of Anthropology, University of Michigan No. 26. Ann Arbor. 
Yerkes, Richard W. 1987 Prehistoric Life on the Mississippi Floodplain: Stone Tool Use, Settlement Organization, and Subsistence Practices at the Labras Lake Site, Illinois. The University of Chicago Press. 